\title{
Plantar pressure image fusion for comfort fusion in diabetes mellitus using an improved fuzzy hidden Markov model
}

Article

Accepted Version

Creative Commons: Attribution-Noncommercial-No Derivative Works 4.0

Li, Z., Wang, D., Dey, N., Ashour, A. S., Sherratt, S. and Shi, F. (2019) Plantar pressure image fusion for comfort fusion in diabetes mellitus using an improved fuzzy hidden Markov model. Biocybernetics and Biomedical Engineering, 39 (3). pp. 742-752. ISSN 0208-5216 doi:

https://doi.org/10.1016/j.bbe.2019.06.007 Available at https://centaur.reading.ac.uk/84495/

It is advisable to refer to the publisher's version if you intend to cite from the work. See Guidance on citing.

To link to this article DOI: http://dx.doi.org/10.1016/j.bbe.2019.06.007

Publisher: Elsevier

All outputs in CentAUR are protected by Intellectual Property Rights law, including copyright law. Copyright and IPR is retained by the creators or other copyright holders. Terms and conditions for use of this material are defined in the End User Agreement. 


\section{CentAUR}

Central Archive at the University of Reading

Reading's research outputs online 


\title{
Plantar pressure image fusion for comfort fusion in diabetes mellitus using an improved fuzzy hidden Markov model
}

\author{
Zairan Li ${ }^{1,2}$, Dan Wang ${ }^{1}$, Nilanjan Dey ${ }^{3}$, Amira S. Ashour ${ }^{4}$, R. Simon Sherratt ${ }^{5}$, and Fuqian Shi ${ }^{6, *}$
}

1. School of Electrical Engineering and Information Engineering, Tianjin University, Tianjin, 300072, PR China

2. Wenzhou Vocational \& Technical College, Wenzhou, 325035, PR China

3. Department of Information Technology, Techno India College of Technology, West Bengal 740000, India.

4. Department of Electronics and Electrical Communications Engineering, Faculty of Engineering, Tanta University, Tanta 31527 Egypt

5. Department of Biomedical Engineering, the University of Reading, RG6 6AY, UK

6. First Affiliated Hospital of Wenzhou Medical University, Wenzhou 325035, China.

*Corresponding authors:

Fuqian Shi \#7B-219, Wenzhou Medical University, Wenzhou, 325035, P.R. China, e-mail: sfq@wmu.edu.cn

+This work is supported by The First Group of Teaching Reform Research Projects in the 13th Five-Year Plan of Higher Education in Zhejiang Province: Development and Practice of Intelligence Technology in Training Mode of Footwear Professionals in Higher Vocational Colleges under Grant No. jg20180589 


\title{
Plantar pressure image fusion for comfort fusion in diabetes mellitus using an improved fuzzy hidden Markov model
}

\begin{abstract}
Diabetes mellitus is a clinical syndrome caused by the interaction of genetic and environmental factors. The change of plantar pressure in diabetic patients is one of the important reasons for the occurrence of diabetic foot. The abnormal increase of plantar pressure is a predictor of the common occurrence of foot ulcers. The feature extraction of plantar pressure distribution will be beneficial to the design and manufacture of diabetic shoes that will be beneficial for early protection of Diabetes mellitus patients. In this research, texture-based features of the Angular Second Moment (ASM), Moment of Inertia (MI), Inverse Difference Monument (IDM), and Entropy (E) have been selected and fused by using the an up-down algorithm. The fused features are normalized to predict comfort plantar pressure imaging dataset using an improved Fuzzy Hidden Markov Model (FHMM). In FHMM, type-I fuzzy set is proposed and Fuzzy Baum-Welch algorithm is also applied to estimate the next features. The results are discussed, and by comparing with other back-forward algorithms and different fusion operations in FHMM. Improved HMMs with up-down fusion using type-I fuzzy definition performs high effectiveness in prediction comfort plantar pressure distribution in an image dataset with an accuracy of $82.2 \%$ and the research will be applied to the shoe-last personalized customization in the industry.
\end{abstract}

Keywords: plantar pressure imaging dataset; image fusion; texture-based feature extraction; fuzzy hidden Markov model; fuzzy Baum-Welch algorithm

\section{Introduction}

Diabetic foot ulcer or gangrene occurs in a certain period of time, and the increase of plantar pressure is the most important factor leading to foot ulcer. Therefore, the detection of plantar pressure in diabetic patients has important clinical significance for the prevention and treatment of diabetic foot. At present, many diabetic hospitals have introduced footwear and insoles with therapeutic correction. The purpose is to reduce the foot pressure of diabetic patients in an area of excessive concentration, reduce related tissue damage and promote ulcer healing, thereby preventing the occurrence of diabetic foot. Foot pressure detection is an advanced technology which is based on the principle of human biomechanics, objective evaluation and prediction of future foot diseases and walking, providing advanced scientific methods of scientific rehabilitation treatment. While in traditional plantar pressure analysis, Image mining-based technologies for plantar pressure research is insufficient. Dynamic reaction of Diabetes mellitus in plantar pressure needs to be addressed, so time series-based analysis can be applied, the target of this research is to apply time-series analysis by using plantar pressure image fusion and so as to produce comfort shoe for Diabetes mellitus in the industry. According to the relevant medical and clinical studies, diabetic foot is most commonly affected by complications. The tissue of diabetes mellitus patients is destroyed by the increased plantar pressure concentration causing plantar capillary occlusion, tissue ischemia gangrene, and plantar foot ulcers. Repeated and sustained mechanical pressure can also lead to aseptic autolysis of tissues, leading to infection, ulceration and the risk of gangrene and amputation [1]. Because diabetes is a most complex condition to manage at present, the incidence of complications caused by diabetes mellitus is high, difficult to manage, and the morbidity rate of disability is high, so diabetes experts suggest that reducing the risk of complications of diabetic foot is particularly important. The increase of plantar pressure is an important risk factor for diabetic foot, so testing plantar pressure has two advantages, at least in preventing diabetic foot: one is that it can predict the occurrence and development of diabetic foot ulcer; the other is that foot shape can be obtained after testing, and a shoe or insole especially suitable for diabetic patients can be made, which can be evaluated by studying foot biomechanics [2][3]. Scientifically balancing plantar pressure using foot medical examination is currently employed to correct foot deformations; improve pain, lesions and joint instability. The use of this special shoe or insole can reduce the possibility of diabetic foot development [4].

In diabetes mellitus, sensory neuropathy makes feet loose their self-protection mechanism and can easy to be injured; motor neuropathy makes intrinsic foot muscle atrophy. As the foot (toe) deformity and metatarsal head protrude, the fiber fat pad of the forefoot is moved forward, the local pressure of forefoot and metatarsal head also is increased, and the pressure ratio of forefoot to hind foot is increased, so callus and pressure ulcers mostly occur in the forefoot. Motor neuropathy also occurs in the forefoot which can lead to abnormal gait, 
limited foot and ankle movement, callus formation, and eventually an ulcer. The results showed that the plantar pressure of diabetic patients was significantly higher than that of normal people, and the ratio of the forefoot to hind foot pressure increased in patients with peripheral neuropathy [5] [6]. Research on plantar pressure of diabetic patients including quantitative evaluation of plantar pressure will be beneficial to making reasonable treatment programs in clinical treatment; combined with other clinical examinations, early detection high-risk groups of diabetic foot will provide corrective insole intervention measures for early protection [7].

Plantar pressure distribution has been researched in recent years. Following the scan devices' improvements, plantar pressure distribution detection technologies have been employed [8-10]. The study of plantar pressure distribution can help alleviate the pressure on patients' foot and the critical feature of plantar pressure will be guidance for producing comfortable shoes [11-13]. The plantar pressure distribution characteristics and technical action are consistent; for technical action of some slight changes, it can be made to change the characteristics of the foot [14-16]. These factors do not significantly change the plantar pressure distribution, but can alleviate and improve the condition because of the use of high heels with cause discomfort, so these factors are necessary to explore the structural design of the shoe sole. Comfort is the key aspect in purchasing footwear, which should be considered during the precision design of the shoe [17] [18]. An improvement in simulating the contact at the plantar surface is necessary. Thus, plantar pressure imaging technologies and the processing of image datasets have recently been developed [19]. Shoe-last customizing system has already been reported by using finite element modeling (FEM) in computer-aided design (CAD) methods [20]. Furthermore, scholars have extended the research into the medical and biomedical fields [21-23].

Image fusion can be used to combine some images into a new image using a specific algorithm. The technology has a basic system, including the main contents of image preprocessing, image fusion algorithm, image fusion evaluation and fusion results. Image preprocessing technology mainly includes two tasks, namely image denoising, image registration, and image fusion algorithm from the initial simple (weighted fusion algorithm and maximum value method) for the development of complex multi resolution algorithm (Pyramid, wavelet method). In addition, image fusion performance evaluation can be subjective or objective evaluation [24]. The system can obtain better performance than that of a subset of the components of the composition [25]. With the artificial intelligence development, artificial immune algorithm (AIA) [26] and the Curvelet transform [27] were used for multi-scale image fusion. Otherwise, fully convolutional network for sensor image fusion [28], evidence-based fusion [29] and Covariance Intersection (CI) fusion [30] were all developed to improve the obtained image.

Before image fusion, the input image is processed by feature extraction, segmentation and matching. The preprocessing generally involves digitization, smoothing, recovery, and enhancement. The fused image is pretreated according to the selected image sensor type and image and the fused target. Recently, there have been several more effective de-noising methods which can effectively preserve the edge information while removing noise effects. Some methods use the idea of partial differential equations, and some methods utilize the wavelet domain hidden Markov model. The idea of choosing a de-noising method is based on the features of the image itself. It is helpful for the subsequent processing to study the appropriate image de-noising method and the appropriate parameters for image de-noising [31-33].

Afterwards, a features extraction process is conducted where texture is an important feature of the image. Texture analysis is an important research content of image understanding, analysis and recognition. Texture can be used to describe the difference of the structure, direction, granularity and regularity of the different regions of the image [34]. The texture contains not only the surface properties or characteristics, but also the extent. It reflects the relation between them and the environment. Suitable feature extraction algorithms for plantar pressure images is important for image mining issue. Khalaf et al. introduced peak plantar pressure (PPP), sample entropy (SamEn) and the 2nd wavelet moment (WM2) features of distribution plantar pressure for detecting diabetic peripheral neuropathy and warrant further investigation [35]. Wang et al. proposed the piecewise linear grayscale transformation on original image, and deployed the time domain mean filter and the maximum value filter for preprocess, then determine the position of the feet in the image by the foot localization algorithm for features extraction [36]. In our previous study, mean square error (MSE), and peak signal to noise ratio (PSNR) were used for evaluating the performance of image segmentation learning algorithms that we proposed -full convolution network (FCN) through the AlexNet platform (FCN-AlexNet$8 \mathrm{~s})[37]$. 
Therefore, the texture features in the expression of gray statistical information can reflect the distribution structure and spatial information. Hence, in the present work texture-based features are extracted to study Fuzzy Hidden Markov Model (FHMM) based comfort prediction. The difficulty is to determine the implicit parameters of the process from observable parameters. These parameters are then used for further analysis, such as pattern recognition. A typical statistical Markov model is presented in which the modeled system is considered as a Markov process with unobserved (hidden) states [38]. HMM is a Markov chain, where the state cannot be observed directly. However, each observation vector is through certain probability density distribution for a variety of conditions. It is composed of a corresponding probability density distribution of a state sequence through the observation vector sequence being observed. Therefore, the HMM can be considered a double stochastic process. Huang et al. [39] proposed model-based character extraction method for HMM in images with non-uniform background case. Thanh et al. [40] used HMM for cancer classification combining with Gene Expression (GE). Zhang et al. [41] applied a fuzzy triplet Markov for Synthetic Aperture Radar (SAR) image segmentation. In addition, thermography images [42], multi-modal medical image [43] and protein sequence identification [44] were processed by using HMM combined with fuzzy set and fuzzy clustering algorithms. Recently Xie, et al. [45] introduced a generalized hidden Markov model (GHMM) for solving the problems of aleatory and epistemic uncertainties. Zhao et al. [46] developed FHMM for image segmentation, and Voronoi tessellation (VT) and hidden Markov random field (HMRF) based fuzzy c-means (FCM) algorithm (VTHMRF-FCM) for texture image segmentation and obtain superior results than other FCM based methods.

Hidden Markov model and fuzzy model technologies also have been widely applied in stock prediction [47], Speech Recognition [48] and signal processing [49]. Ferhat et al. [50] introduced an improved HMM for automatic recognition of gait phases; Juri et al. [51] addressed a novel HMM distributed classifier for the detection of gait phases; dynamic gait researches have been developed recently for shoe product industry [52]. Accordingly, the HMM using type-I fuzzy set was proposed in this work through a definition on fuzzy set for parameters and status. Thus, the proposed model was named FHMM.

\section{Methodology}

\subsection{Plantar pressure data acquisition}

10 patients with type 2 diabetes mellitus ( 5 male, 5 female) of 35-55 ages were involved the test using the footscan 7.0 system. The clinical diagnostic criteria were HbA1c is " $>6.5 \%$ ", fasting blood glucose FPG is "> $7.0 \mathrm{mmol} / \mathrm{L}$ "; an empty stomach is defined as no calorie intake for at least eight hours; blood sugar is "> $11.1 \mathrm{mmol} / \mathrm{L}$ " at 2 hours of oral glucose tolerance test; in patients with typical symptoms of hyperglycemia or hyperglycemia crisis; and random blood sugar with " $>11.1 \mathrm{mmol} / \mathrm{L}$ ". In the choice of the way of boarding, taking steps on the way is adopted; in the choice of test times, "take steps" is to collect 4-6 times of data. In the static balance test, control of test time is under four states (double opening, double closing, single opening and single closing). Standing on two feet is usually 20 seconds, standing on one foot is usually 10 seconds. By starting the footscan 7.0 system software and registering the identity of the subjects, imaging database and personnel records are created; by importing the relevant parameters, starting the real-time detection power supply, and instructing the subjects to walk into the force plate by three times, the system software acquires the foot pressure imaging subset in real time, and stores the data automatically until the end of the experiment. The operators select the dynamic model to obtain the change process of plantar pressure. Table 1 shows the details of the parameters of the scanning device in the proposed system while acquiring the planar pressure distribution imaging dataset using the RS-Scan Footscan 7.0 software system (as shown in Fig.3)..

Table 1. The parameters of plantar pressure measurement system

\begin{tabular}{|c|c|c|c|}
\hline Items & Measurement & Items & Measurement \\
\hline Area & $40 * 50 \mathrm{~cm} 2$ & Total sensors & 4096 \\
\hline Numbers of sensor & 4 per cm2 & Sampling Hz & $125-300 \mathrm{~Hz}$ \\
\hline Size of each sensor & $0.5 * 0.7 \mathrm{~cm} 2$ & Analog channels & 16 \\
\hline Image resolution & 12 bits & Entry level & $2 \mathrm{~m}$ \\
\hline
\end{tabular}

Furthermore, the system can also calculate the pressure information in different zone (shown in Fig. 2). Fig 3 showed time series based plantar pressure images under comfort shoes. 

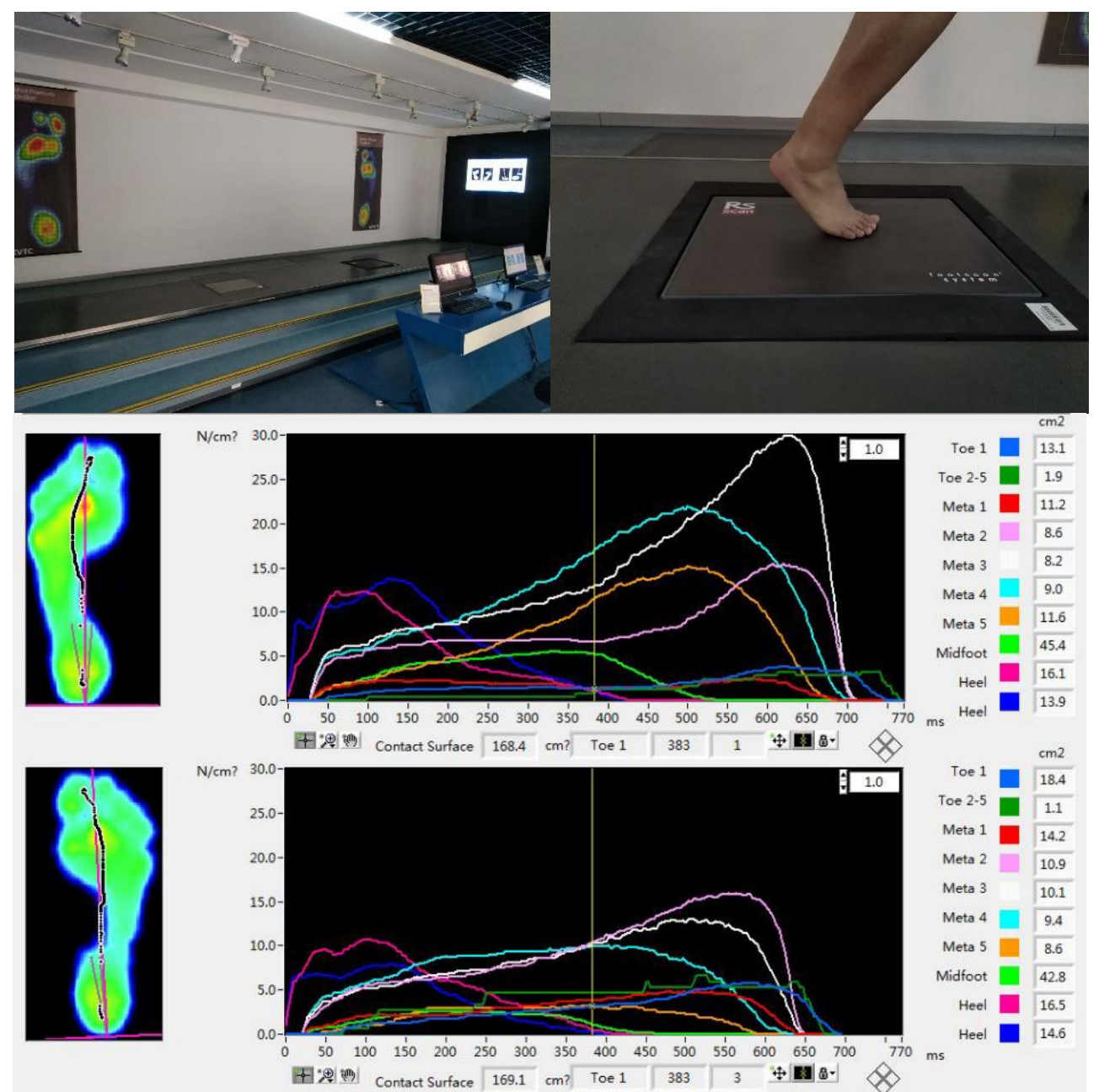

Figure 2. Foot Scan system by RS-scan for measuring plantar pressure and imaging experiment

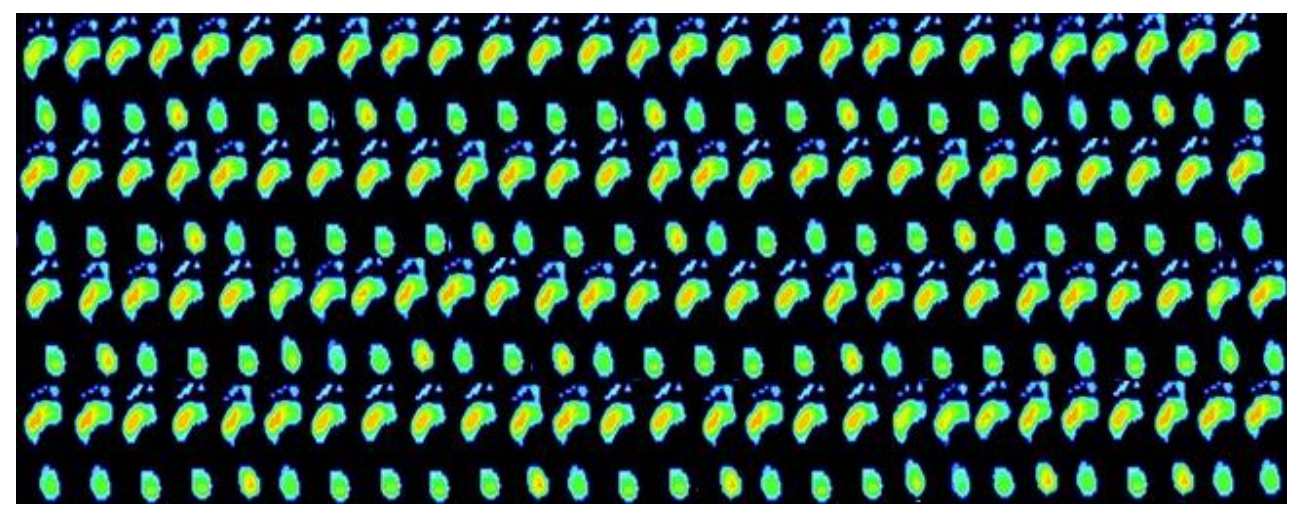

Figure 3. Time-series based plantar pressure images

\subsection{Texture based features}

Gray-level Co-occurrence Matrix (GLCM) is used for image feature calculation. The co-occurrence matrix is calculated based on gray level of image to represent some image texture feature. The GLCM reflects the comprehensive information about the image direction, adjacent interval and amplitude of variation, which is the foundation of image analysis of local patterns and arrangement rules. Out of 14 texture features based on GLCM, only 4 features (Angular Second Moment, Moment of Inertia, Inverse Difference Monument, and 
Entropy) are uncorrelated. Suppose that $(x, y)$ is a point's coordinates in plantar pressure image, $x \in\{1,2, \ldots, m\}, y \in\{1,2, \ldots, n\}$, gray level is $L$, and the nearby point is $(x+\Delta x, y+\Delta y), \Delta x>0$, $\Delta y>0, f(x, y)$ is the value of point. The gray value can be calculated by $g_{\Delta}(f(x, y))=g(f(x, y))-g(f(x+\Delta x, y+\Delta y))$, where, $g_{\Delta}$ is gray difference. Assume there are $m$ levels, count $g_{\Delta}$ in each $m$, then the probability on each $g_{\Delta}$ can be acquired as $p(i),(i=1, \cdots, m)$. GLCM extracts the texture by using gray and gradient synthetic information. The process is like the GLCM. The Sobel operator can be applied to $f(x, y)$ in order to obtain the gray image $g(x, y)$ and be discretized to be $G(x, y)$. Let the new gray level be $L_{g}$, then new gray is calculated by:

$$
G(x, y)=\frac{g(x, y)-\min (g(x, y)}{\max (g(x, y))-\min (g(x, y))}\left(L_{g}-1\right)
$$

where $H_{i j}$ is defined as number of $\{(x, y) \mid f(x, y)=o, G(x, y)=j\}$. Normalized $\mathrm{H}_{i j}$ as follows:

$$
P_{i j}=\frac{H_{i j}}{\sum_{i=0}^{L-1} \sum_{j=0}^{L-1} H_{i j}}
$$

(1) Angular Second Moment (ASM)

$$
A S M=\sum_{i=0}^{L-1} \sum_{j=0}^{L_{g}-1} P_{i j}^{2}
$$

(2) Moment of Inertia (MI)

$$
M I=\sum_{i=0}^{L-1} \sum_{j=0}^{L_{g}-1}(i-j)^{2} P_{i j}
$$

(3) Inverse Difference Monument (IDM)

(4) Entropy (E)

$$
I D M=\sum_{i=0}^{L-1} \sum_{j=0}^{L_{g}-1} \frac{1}{1+(i-j)^{2}} P_{i j}
$$

$$
E=-\sum_{i=0}^{m} p(i) \log _{2}(p(i))
$$

The principals for texture features selection are: i) for the window size $N$ 's selection, a large value will result in a large amount of calculation and storage, but if it is too small, it will not contain complete texture information. In general, when the image size is determined, the calculation window is then determined, unless the image is processed in blocks, or the Region of Interest (ROI); and then the GLCM are extracted. ii) For gray level $L$ selection, the gray level determines the computational scale of GLCM; and reduces the gray level to improve the calculation speed and reduce the storage space requirements. iii) In the selection of the distance $D$, the co-occurrence matrix changes rapidly with the distance in fine texture, while in the rough texture it varies slowly with distance. For smooth textures with larger distances, a smaller distance for a rough texture will lead to better results. vi) For direction selection, there are four kinds of values of $0,45,90$, and 135 degrees. Different theta can examine different textures, different co-occurrence matrix generated in different theta, by considering the direction, the mean values of four directions of gray level co-occurrence texture features matrix are calculated, then each direction eigenvalue is the final texture component. v) The entropy is used to measure the amount of information and reflects the disorder or the complexity of the texture of the image. vi) IDM directly measures the local uniformity of digital images and the larger the value of the deficit, the more consistent the image is.

\subsection{Fusion operation}

The images of plantar pressure dataset are time series based, and the image fusion operation makes more effect on the data set to get key points in images and then for comfort prediction. In this study, up-down 
fusion was applied based our previous studies [19]. Supposed that the matrices $A$ and $B$ are two time-series based plantar pressure images, and the fusion algorithm is "Up-Down" referring [53] [54].

\subsection{Fuzzy Hidden Markov Model for comfort prediction}

Assume the observation sequence $O=\left\{O_{1}, O_{2}, \cdots O_{t}\right\}$ and the model parameter $\lambda=(A, B, \pi)$. The probability of an observation sequence can be effectively calculated, and then make a relevant assessment of the HMM for the next steps. For example, there are some HMMs with different model parameters, by giving the observation sequence $O$, the problem is which HMM model is most likely to generate the observation sequence. Usually the forward algorithm can be used to calculate the probability that each HMM produces a given observation sequence $O$, and then choose the optimal HMM model. Afterward the decoding problem is considered to find a sequence of implicit states is an optimal problem in some sense. Among these problems, hidden states in the Markov model were proposed, which means the system cannot be directly observed, but are more valuable, and are usually searched using the Viterbi algorithm. A practical example of such a problem is the Chinese word segmentation, that is to divide a sentence into its composition, and the HMM can be constructed to find the most likely correct word segmentation method. Finally, the learning process is considered, where the model parameter $\lambda=(A, B, \pi)$ of the HMM is unknown. Thus, adjusting these parameters so that the probability of the observation sequence $O$ is as large as possible becomes essential. It is usually solved using the Baum-Welch algorithm and the Reversed Viterbi algorithm.

\subsubsection{Forward algorithm}

As above mentioned, HMM is used to solve three problems: evaluation, decoding, and learning. In the HMM, the forward algorithm is used for evaluation, while the Viterbi algorithm is used for decoding. Supposed that the number of hidden states is $N$, and the number of observed sequences is $T$. Obviously, for a certain observation sequence, the hidden state sequence has a common situation in $N^{T}$. If the probability is obtained by the exhaustive method, the complexity is $N^{T}$. A recursive approach to reduce the complexity of the problem can be used, which is the forward algorithm, Fig.1 illustrates the framework of the forward algorithm of HMM.

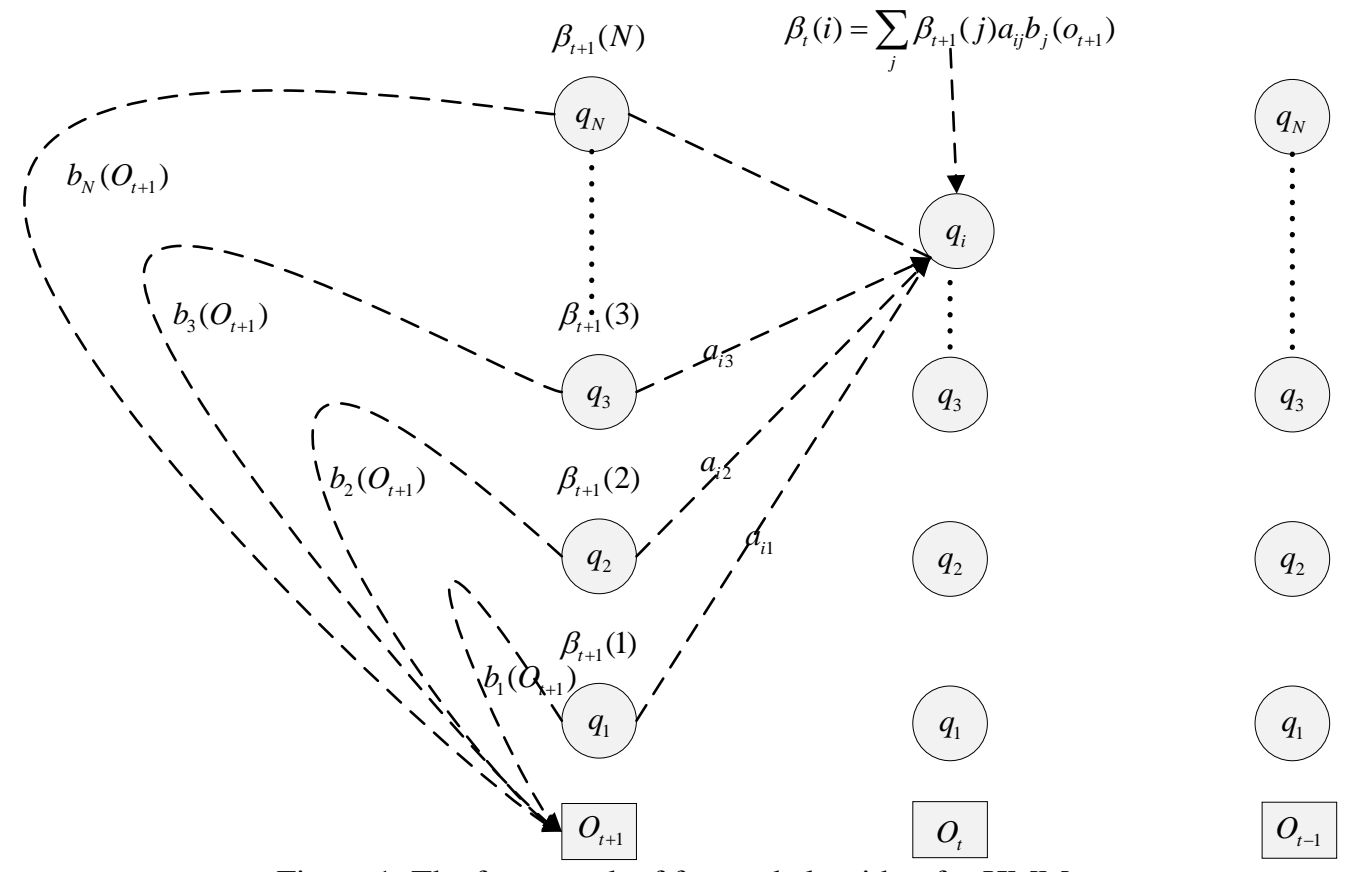

Figure 1. The framework of forward algorithm for HMM 
At time $t$, the probability of state $j$ is $a_{t}(j)=P\left(O \mid q_{j}\right) \times P$ (the path of all arrival state $j$ at time $t$ ), then proceed with forward recursion as follows:

(1) Initialization: Calculate the local probability at $t=1, a_{1}(j)=\pi(j) \times b_{j}\left(O_{1}\right), 1 \leq j \leq N$.

(2) Recursion: Calculate the local probability when $t>1, a_{t}(j)=\sum_{i=1}^{N} a_{(t-1)}(j) \times a_{i j} \times b_{j}\left(O_{t}\right)$, $1 \leq j \leq N, 1 \leq t \leq T$.

(3) Terminate at $t=T$, where $a_{(t-1)}(j)$ represents the local probability generated at time $t-1, a_{i j}$ represents the probability of transitioning from state $i$ at time $t-1$ to state $j$ at time $t$, and $b_{j}\left(O_{t}\right)$ represents observation from state $j$. The probability of the value $O_{t}$. Algorithm 1 shows the forward algorithm of HMM.

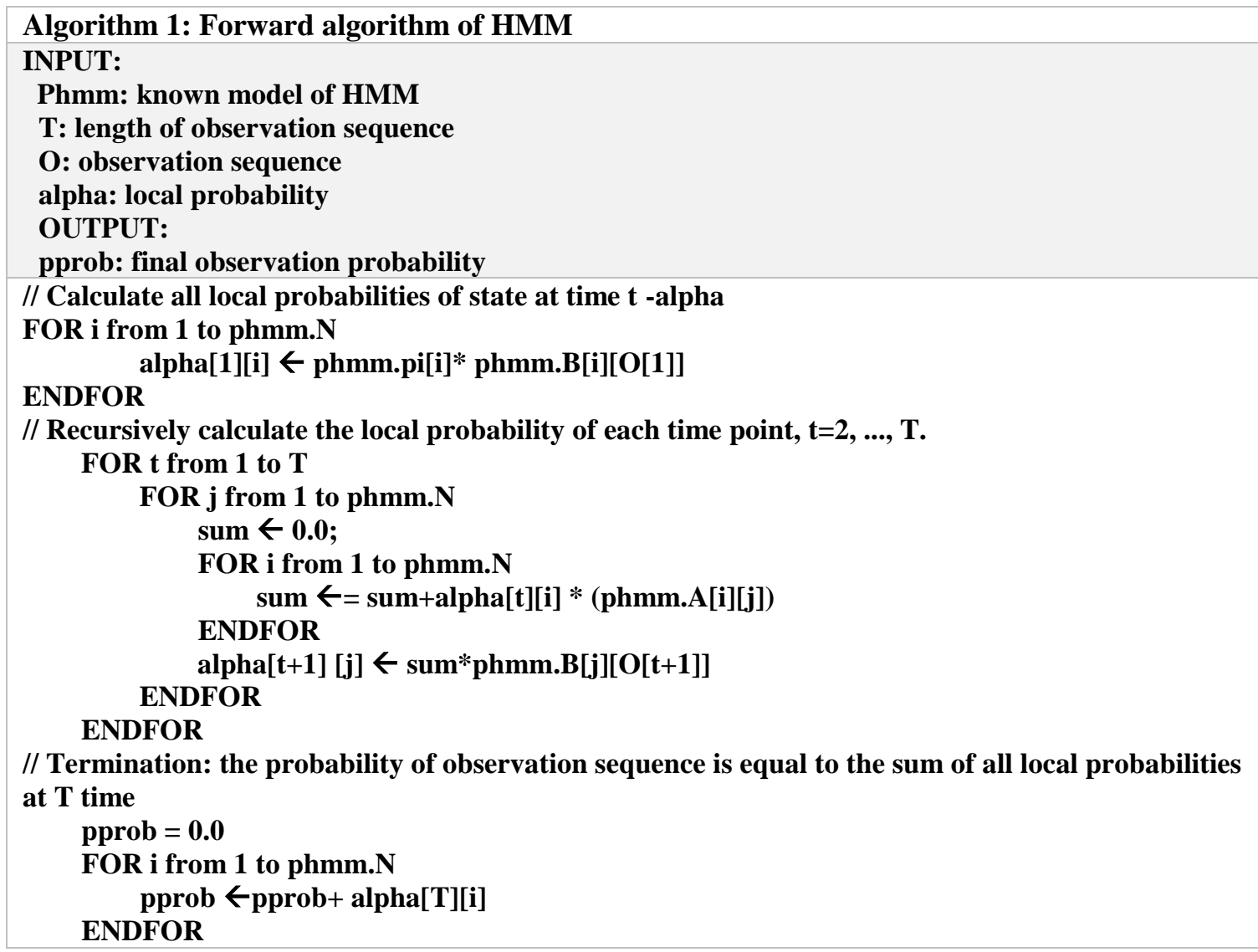

\subsubsection{Baum-Welch estimation in Hmm}

The first issue is decoding to find the best hidden state sequence for an observation sequence. For each hidden state sequence $Q$, calculate $P(O \mid Q)$ and select one of the cases with the highest probability. The complexity is $N^{T}$, and the Viterbi algorithm is used for recursive calculation to reduce the complexity. By defining the probability of being in state $j$ at time $t$ is $V_{t}(j)$. Unlike the forward algorithm, the Viterbi algorithm finds the probability of the most probable path to a certain state at time $t$, instead of all paths. The sum of the probabilities can be calculated as the steps as follows. 
Step 1 Initialization, when $t=1$, it is obvious that the most probable path to a certain state is nonexistent, that is, the local probability at time $t=1$ is $V_{i}(j)=\pi(j) \times b_{j}\left(O_{1}\right), 1 \leq j \leq N$

Step 2 Recursion by calculating the local probability when $t>1$, and

$$
V_{t}(j)=\operatorname{Max}_{1 \leq i \leq N}\left(V_{t-1}(j) \times a_{i j} \times b_{j}\left(O_{t}\right)\right.
$$

Step 3 Terminates until $t=T$.

The Viterbi algorithm introduces a reverse pointer, performs Viterbi backtracking, and records the best state. The argmax operator is used to calculate the index $j$ that maximizes the value of the expression in parentheses. Formally, we have that:

$$
b_{t}(j)=\arg \max _{1 \leq i \leq N}\left\{V_{t-1}(j) \times a_{i j} \times b_{j}\left(O_{t}\right\}, 1 \leq j \leq N, 1<t<T\right.
$$

When the entire calculation process is completed, the most probable state is first found at the end time, and then backtracked to the time $t=1$ by the backward pointer, so that the state sequence on the backtracking path is the most likely hidden state sequence (See the Algorithm 2).

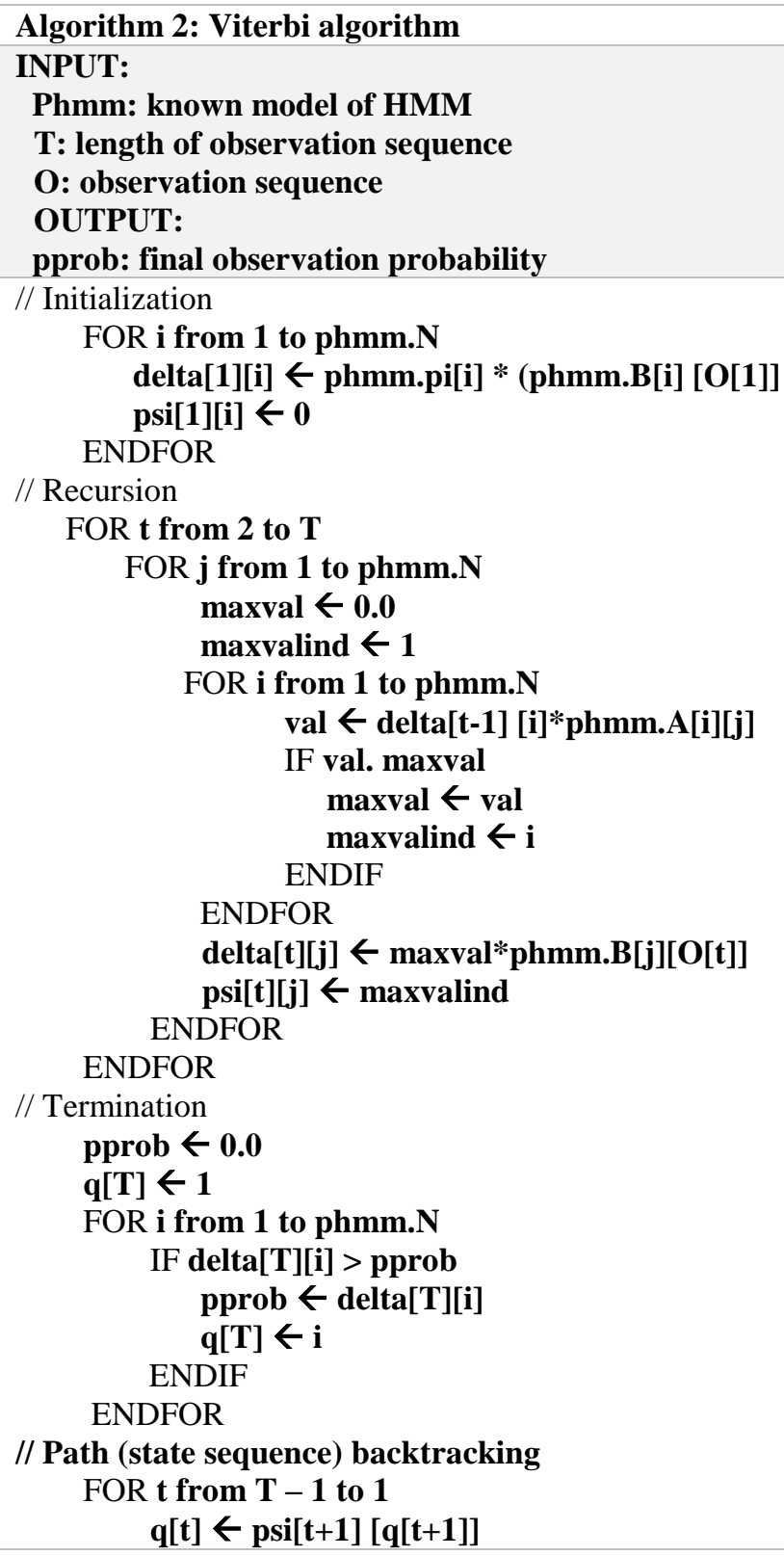




\section{ENDFOR}

\subsubsection{Proposed Fuzzy HMM}

In this work, firstly, parameters of FHMM were defined. Suppose that fuzzy set can be formalized as $\hat{A}$; and fuzzy HMM is presented as:

$$
\hat{\mu}=(\hat{S}, \hat{O}, \hat{A}, \hat{B}, \hat{\pi}, \hat{F})
$$

where $S^{o}$ is initial fuzzy measure of status; $\hat{O}$ is a finite set of observe and in time $t, \hat{A}$ is transmission matrix of status $B=\left\{b_{i j}(k)\right\}$ is a set of probability of observe $k$ from status $\hat{S}_{i}$ to $\hat{S}_{j}, \hat{\pi}$ is fuzzy density function; $\hat{F}$ is fuzzy measured final status set. Using fuzzy presentation for Baum-Welch algorithm, the process is updated following fuzzification of parameters estimation for the FHMM. The process is:

$$
\begin{aligned}
E_{S_{k}}(c) & =\frac{1}{P(O)} \sum_{i \mid O_{i}=c} f_{S_{k}}(i) \rho_{i}\left(S_{k}, .\right) b_{S_{k}}(i) \\
E_{O_{k}}(c) & =\frac{1}{P(O)} \sum_{i \mid O_{i}=c} f_{O_{k}}(i) \rho_{i}\left(O_{k}, .\right) b_{O_{k}}(i) \\
A_{S_{k} S_{k+1}} & =\frac{1}{P(O)} \sum_{i} f_{S_{k}}(i) a_{S_{k} S_{k+1}} \rho_{i}\left(S_{k}, S_{k+1}\right) e_{S_{k+1}}\left(x_{i}+1\right) b_{S_{k+1}}(i+1) \\
A_{S_{k} O_{k}}= & \frac{1}{P(O)} \sum_{i} f_{S_{k}}(i) a_{S_{k} O_{k}} \rho_{i}\left(S_{k}, O_{k}\right) e_{O_{k}}\left(x_{i}+1\right) b_{O_{k+1}}(i+1) \\
A_{S_{k} D_{k+1}}= & \frac{1}{P(O)} \sum_{i} f_{S_{k}}(i) a_{S_{k} D_{k+1}} \rho_{i}\left(S_{k}, D_{k+1}\right) b_{D_{k+1}}(i)
\end{aligned}
$$

where $D$ is initial fuzzy presented status, $\rho$ is a fuzzy density function can be calculated by Algorithm 3 .

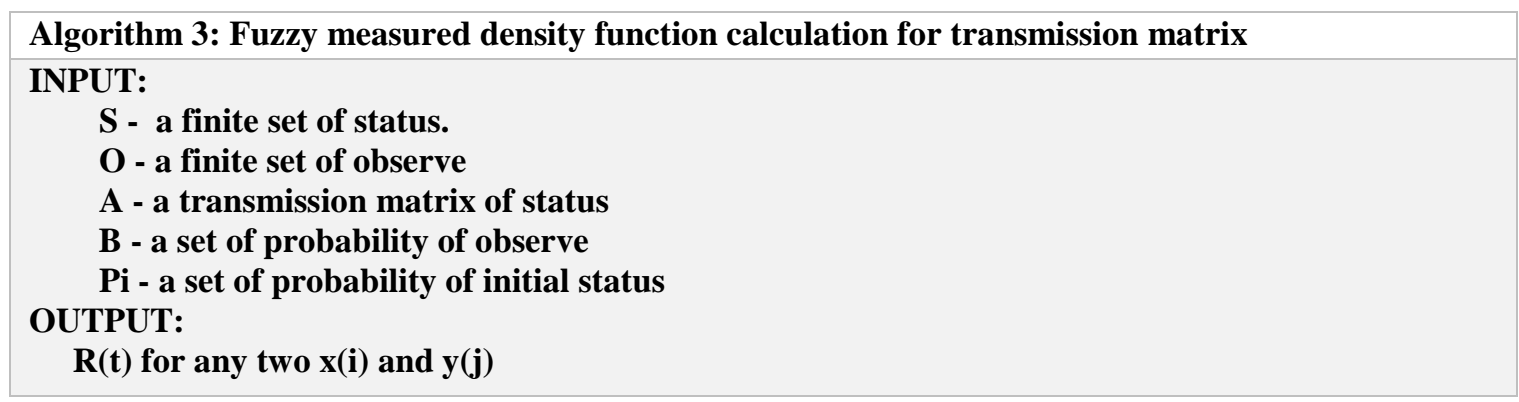




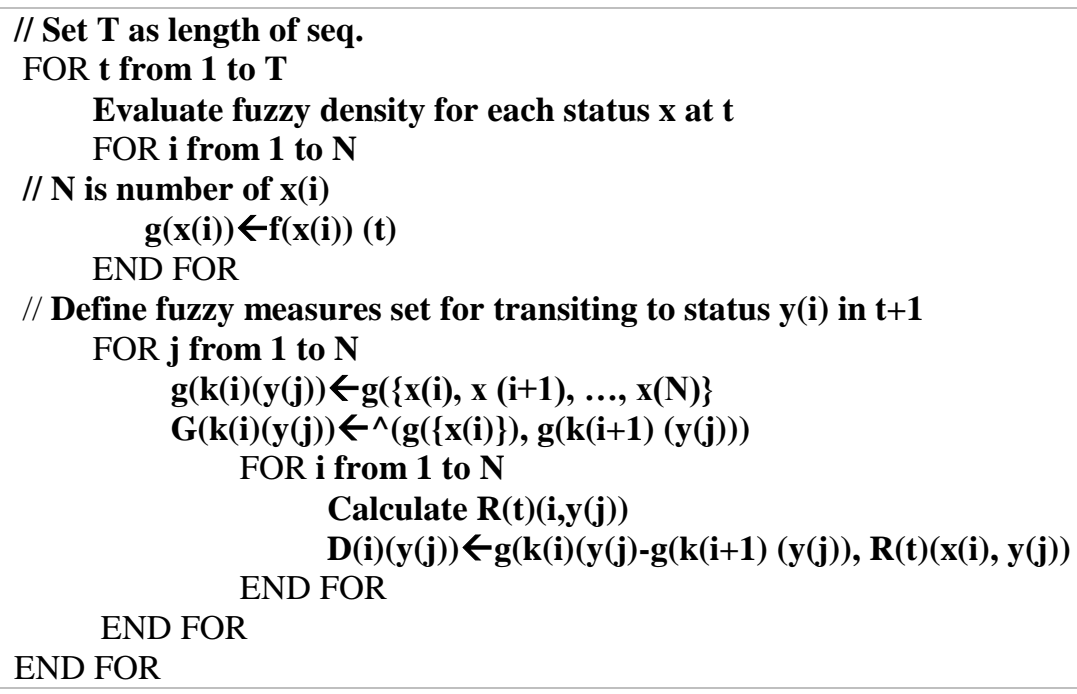

The pseudo-code of fuzzy Baum-Welch estimation in FHMM is in Algorithm 4 as follows:

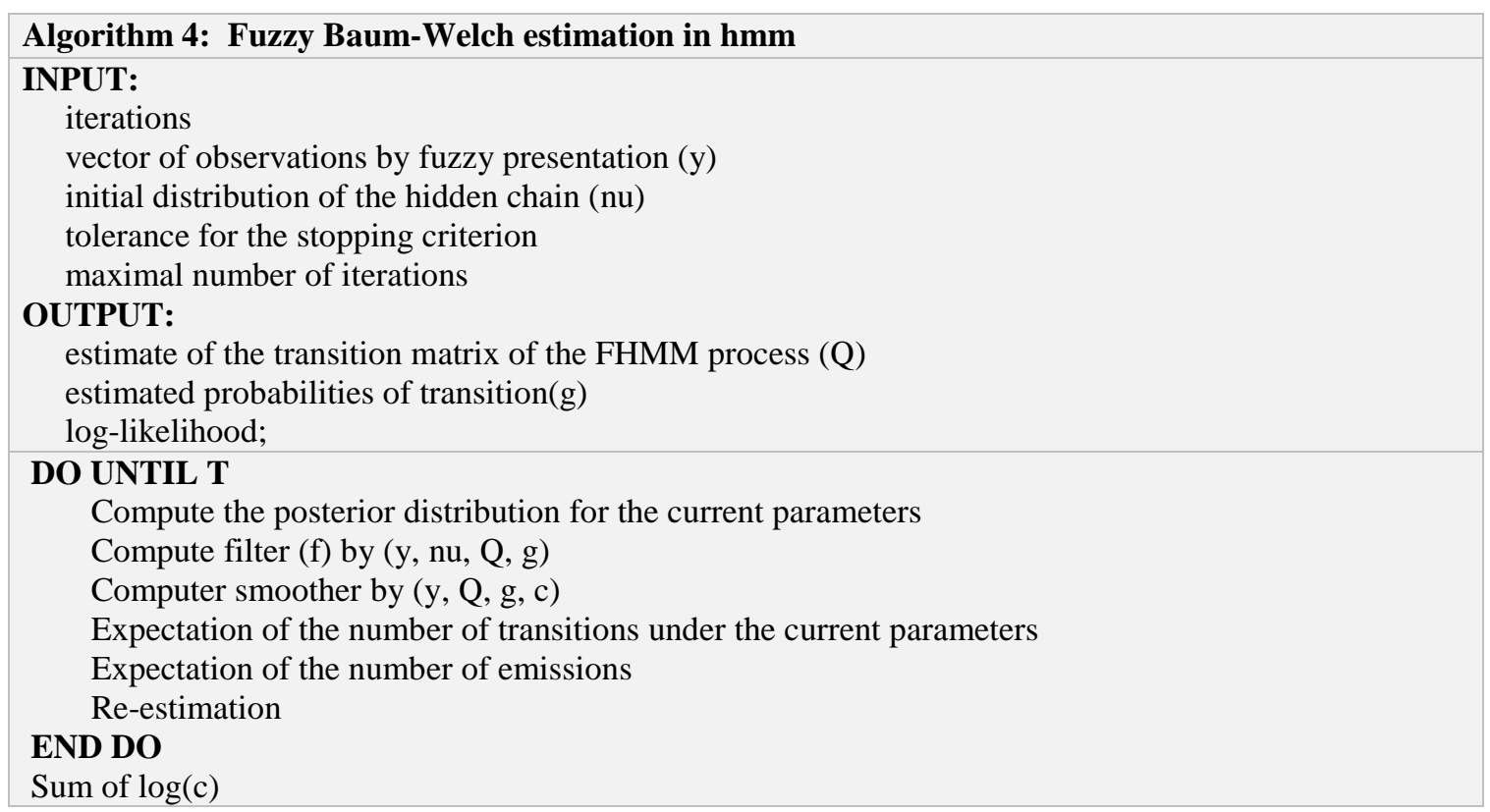

\section{Results and discuss}

In the current work, the analysis was performed on a Windows $10 \mathrm{PC}$ using a 64-bit AMD Athlon(tm) II X4 631 Quad-Core CPU at 2.6GHz running MATLAB R2017a.3.1 ResultsThe redundant information provided by multiple sensors can improve the image accuracy and reliability, but for time series-based image set, the fused image contains more comprehensive and rich information as compared to single image. In the experiment, eight images of the same testee's in different test subjects were selected for fusion. For simplicity, two sets of testee's plantar pressure images were calculated. Table 2 illustrated the first test of testee's and Table 3 is from second test.

Table 2. The first test of testee's features calculation on eight images

\begin{tabular}{lllllllll}
\hline Features & Img11 & Img12 & Img13 & Img14 & Img15 & Img16 & Img17 & Img18 \\
\hline ASM & 0.4075 & 0.4214 & 0.3944 & 0.3644 & 0.4788 & 0.3733 & 0.3878 & 0.4844 \\
MI & 0.6566 & 0.6565 & 0.6440 & 0.5876 & 0.5467 & 0.5432 & 0.4897 & 0.6434 \\
\hline
\end{tabular}




\begin{tabular}{lllllllll}
\hline IDM & 0.1765 & 0.1876 & 0.1222 & 0.1678 & 0.1976 & 0.2009 & 0.2322 & 0.2127 \\
E & 0.8978 & 0.6899 & 0.7893 & 0.8766 & 0.7876 & 0.8754 & 0.7987 & 0.8656 \\
\hline
\end{tabular}

(Note: Angular second moment -ASM, Moment of inertia -MI, Inverse difference monument -IDM, Entropy -E.)

Table 3. The second test of testee's features calculation on eight images

\begin{tabular}{lllllllll}
\hline Features & Img21 & Img22 & Img23 & Img24 & Img25 & Img26 & Img27 & Img28 \\
\hline ASM & 0.3444 & 0.4323 & 0.5432 & 0.5322 & 0.3544 & 0.3788 & 0.4322 & 0.4312 \\
MI & 0.3898 & 0.3433 & 0.5677 & 0.5233 & 0.4899 & 0.42233 & 0.5433 & 0.4567 \\
IDM & 0.2788 & 0.3019 & 0.3221 & 0.21291 & 0.2245 & 0.2865 & 0.2238 & 0.2182 \\
E & 0.7896 & 0.7698 & 0.8743 & 0.7568 & 0.8777 & 0.8973 & 0.8650 & 0.7908 \\
\hline
\end{tabular}

And fusion operation using up-down was shown Fig. 4. The features of Fig 4 were calculated in Table 4.

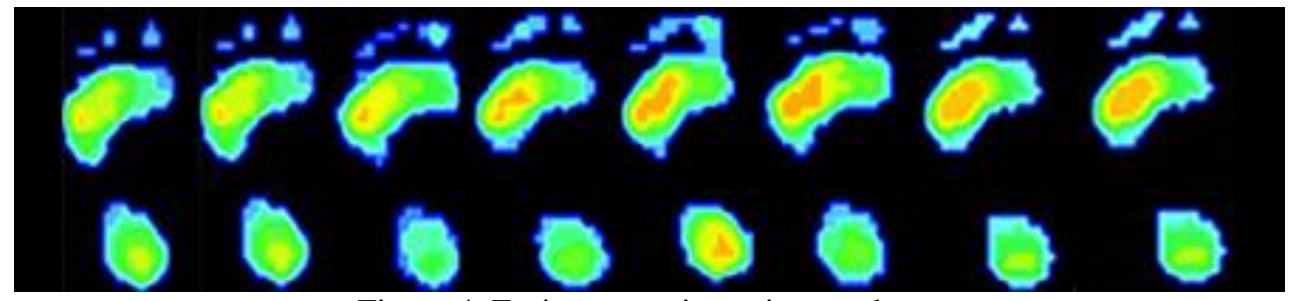

Figure 4. Fusion operation using up-down

Table 4. Fused images' feature calculation

\begin{tabular}{lllllllll}
\hline Features & Img1 & Img2 & Img3 & Img4 & Img5 & Img6 & Img7 & Img8 \\
\hline ASM & 0.7655 & 0.7666 & 0.5664 & 0.5210 & 0.6230 & 0.6743 & 0.4320 & 0.4565 \\
MI & 0.4743 & 0.45677 & 0.4534 & 0.5023 & 0.5430 & 0.5784 & 0.5094 & 0.4903 \\
IDM & 0.1200 & 0.1245 & 0.1601 & 0.2322 & 0.2765 & 0.2654 & 0.2190 & 0.1121 \\
E & 0.8433 & 0.9061 & 0.7988 & 0.8234 & 0.8789 & 0.7666 & 0.7680 & 0.8232 \\
\hline
\end{tabular}

All these textures-based features calculation was normalized in $(0,1]$. Set initial status as (ASM, MI, IDM, E) for every 8 fused images, observe vector used fuzzy evaluation for comfort using fuzzy linguistic variables which defined as \{very uncomfortable, uncomfortable, median, comfortable, very comfortable\}, and presented as fuzzy number set as $\{\hat{1}, \hat{3}, \hat{5}, \hat{7}, \hat{9}\}$. Additionally, the transmission matrix $A$ was calculated by statistics method based on those fuzzy numbers, the probability between each status of $i$ and $j$ also can be also calculated based on fuzzy numbers. As from the fuzzy Baum-Welch estimation algorithm proposed in Algorithm $3, \quad$ set iterations $n=500, n u=[0,1], Q=[0.7,0.3 ; 0.2,0.8], \quad$ and

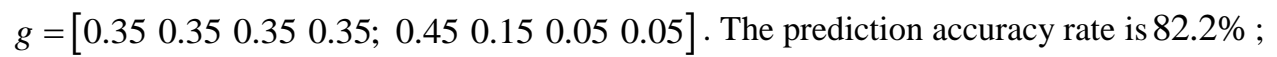

3.2 Discuss

FHMM using different fusion operations was deployed for comparing analysis with typical fusion methods, such as max-min, mean-mean, Gaussian as listed in Table 5.

Table 5. The accuracy rate of the same FHMM using different fusion operations

\begin{tabular}{ll}
\hline Fusion operations & Accuracy Rate \\
\hline MAX-MIN [54] [55] & $\mathbf{0 . 7 5 7 3}$ \\
MEAN-MEAN [25] & $\mathbf{0 . 7 1 0 2}$ \\
GAUSSIAN [56] & $\mathbf{0 . 7 1 4 4}$ \\
UP-DOWN (this research) & $\mathbf{0 . 8 2 2 0}$ \\
\hline
\end{tabular}

For performing the prediction model in this work, different typical HMMs using up-down fusion were addressed. The proposed FHMM model achieved high accuracy rate for prediction. In which, DHMM is discrete hidden Markov model; DDBHMM means duration distribution based hidden Markov; CRF denoted conditional random field; MEHMM is maximum entropy hidden Markov model; and MRF is Markov random filed. For the results, FHMM using up-down fusion deployed high performance in this case. 
Table 6. Different typical HMMs using up-down fusion for prediction

\begin{tabular}{ll}
\hline Typical HMMs & Accuracy Rate \\
\hline DHMM [57] & 0.6922 \\
DDB-HMM [58] & 0.6954 \\
HMM [59] & 0.7144 \\
CRF [60] & 0.7510 \\
MEHMM [61] & 0.7120 \\
MRF [62] & 0.6965 \\
FHMM (this research) & 0.8220 \\
\hline
\end{tabular}

\section{Conclusion}

The formation of diabetic foot ulcer is directly related to the repeated high pressure on the ulcer site during standing or walking. Foot injury caused by improper shoe selection; repeated pressure stimulation caused by improper shoes, socks and pads, which affected local circulation, caused skin damage, epidermal keratosis, worsened local ischemia, damage, corns, ulcers, gangrene. Diabetic footwear is a new type of healthy footwear specially designed for the protection of diabetic foot, its sole formation mainly depends on the characteristics of the sole pressure image. The study of plantar pressure distribution is very important to the process of producing comfortable shoes for people with a side variety of medical conditions. From an image set of plantar pressure, and through analyzing features of plantar pressure images such as texture-based features of ASM, MI, IDM, and E, the proposed FHMM model achieved the superior accuracy rate in comparison with different typical HMMs using up-down fusion for prediction. The primary research contributions of the work include:

(1) Image fusion technology was used to fuse the same subjects in different experiments to investigate if the image contains more content in the image preprocessing stage.

(2) Four unrelated features separated from many image features are used as input states of FHMM, and the construction of observation and evaluation sets by means of fuzzy numbers is used to achieve better results.

(3) An improved HMM was compared under different fusion methods, which was proven to be an effective use of our method. In future works, the state presentation of FHMM for plantar pressure imaging dataset and the fuzzy number's definition also needs to be improved.

In future works, more datasets need to be collected through footscan system, feature selection, feature extraction algorithms for plantar pressure images are also need to be improved.

\section{Compliance with ethical standards}

Conflict of interest: None declared

Ethical approval: Not required

\section{Acknowledgement}

This work is supported by XXX.

\section{References}

[1] Cao, L, Dey, N, Ashour, AS. Simon F, Sherratt RS, Wu L, Shi F, Diabetic plantar pressure analysis using image fusion, Multimed Tools Appl, 2018, https://doi.org/10.1007/s11042-018-6269-x

[2] Liu S, He C, Cai Y, Xing Q, Guo Y, Chen Z, Su J, Yang L. Evaluation of negative-pressure wound therapy for patients with diabetic foot ulcers: Systematic review and meta-analysis, Therapeutics and Clinical Risk Management, 2017; 13, 533-544

[3] Fernando ME, Crowther RG. Lazzarini PA, Sangla KS, Wearing S, Buttner P, Golledge J, Plantar pressures are higher in cases with diabetic foot ulcers compared to controls despite a longer stance phase duration, BMC Endocr Disord, 2016; 16: 51

[4] Falzon B, Formosa C, Camilleri L, Gatt A, Duration of Type 2 Diabetes is a Predictor of Elevated Plantar Foot Pressure, The Review of Diabetic Studies, 14(4):372-380. 
[5] Andrew KB, Jamie JA, Karl BL, Hylton BM, The relationship between foot posture and plantar pressure during walking in adults: A systematic review, Gait \& Posture, 62, 56-67, 2018

[6] Chen H, Cao L, Li Z, Hemanth DJ, Wu L, Albuquerque DHC, Shi F. Evaluation on diabetic plantar pressure data-set employing auto-segmentation technologies, Neural Comput \& Applic (2018). https://doi.org/10.1007/s00521-018-3838-x

[7] Arts ML, Bus SA. Twelve steps per foot are recommended for valid and reliable in-shoe plantar pressure data in neuropathic diabetic patients wearing custom made footwear, Clin Biomech (Bristol, Avon). 26(8):880-4, 2011

[8] Razak AH, Zayegh A, Begg RK, Wahab Y. Foot Plantar Pressure Measurement System: A Review, Sensors, 2012; 12(7): 9884-9912

[9] Tong JW, Kong PW. Reliability of footprint geometric and plantar loading measurements in children using the Emed (R)M system, Gait Posture. 2013; 38(2):281-286

[10] Silva AH, Sampath WH, Sameera NH, Amarasinghe YW, Mitani A. Development of a novel telecare system, integrated with plantar pressure measurement system, Informatics in Medicine, 12, 2018, 98-105

[11] Wang D, Li Z, Dey N, Ashour AS, Sherratt RS, Shi F. Case-Based Reasoning for Product Style Construction and Fuzzy Analytic Hierarchy Process Evaluation Modeling Using Consumers Linguistic Variables, IEEE Access, 2017; 5: 4900-4912.

[12] Kornfeind P, Eckl M, Baca A. A single step analysis of plantar pressure distribution in tennis specific movements, Journal of Human Sport and Exercise, 2018; https://doi.org/10.14198/jhse.2019.142.14

[13] Naraghi R, Slack-Smith L, Bryant A. Plantar Pressure Measurements and Geometric Analysis of Patients With and Without Morton's Neuroma. Foot \& Ankle International, 2018(2): AID: 1071100718766553.

[14] Lipsitz LA, Lough M, Niemi J, Travison T, Howlett H, Manor B. A shoe insole delivering sub-sensory vibratory noise improves balance and gait in healthy elderly people, Archives of Physical Medicine and Rehabilitation, 2015; 96(3):432-439.

[15] Nishiwaki T, Nonogawa M. Application of topological optimization technique to running shoe designing, Procedia Engineering, in proc. The Impact of Technology on Sport VI 7th Asia-Pacific Congress on Sports Technology, APCST2015, 2015;.112, p.314-319.

[16] Juanita W, Hank W, Sam A, Shapiro R, Walker J. Foot pressure analysis using the emed in typically developing children and adolescents: A summary of current techniques and typically developing cohort data for comparison with pathology, The Foot, 2018; 37, 28-37,

[17] Luximon A, Luximon Y. Shoe-last design innovation for better shoe fitting, Computers in Industry, 2009; 60 (8): $621-628$.

[18] Rangra P, Santos D, Coda A, Jagadamma K. The Influence of Walking Speed and Heel Height on

Peak Plantar Pressure in the Forefoot of Healthy Adults: A Pilot Study, Clinical Research on Foot \& Ankle, 2017; 5(2): 100239-6

[19] Wang D, Li Z, Cao L, Balas VE, Dey N, Ashour AS, McCauley P, Shi F. Image Fusion Incorporating Parameter Estimation Optimized Gaussian Mixture Model and Fuzzy Weighted Evaluation System: A Case Study in Time-Series Plantar Pressure Data Set, IEEE Sensors Journal, 2017; 17 (5): 1407 - 1420.

[20] Franciosa P, Gerbino S, Lanzotti A, Silvestri L. Improving comfort of shoe sole through experiments based on cad-fem modeling, Medical Engineering \& Physics, 2013; 35 (1):36 - 46.

[21] Horsak B, Heller M, Baca A. Muscle co-contraction around the knee when walking with unstable shoes. Journal of Electromyography and Kinesiology, 2015; 25 (1): 175 - 181.

[22] Souissi H, Zory R, Bredin J, Gerus P. Comparison of methodologies to assess muscle co-contraction during gait, Journal of Biomechanics, 2017; 57: 141-145.

[23] Jacobs DA, Ferris DP. Estimation of ground reaction forces and ankle moment with multiple, low-cost sensors, Journal of NeuroEngineering and Rehabilitation, 2015; 12, 90

[24] W Xu, Yu J. A novel approach to information fusion in multi-source datasets: A granular computing viewpoint, Information Sciences, 2017; 378: 410-423.

[25] Liu Z, Blasch E, John V. Statistical comparison of image fusion algorithms: Recommendations, Information Fusion, 2017; 36: 251-260.

[26] Lu Z, Xiang Q, Xu L. An application case study on multi-sensor data fusion system for intelligent process monitoring, Procedia CIRP, in: Variety Management in Manufacturing, Proceedings of the 47th CIRP Conference on Manufacturing Systems. 2014; 17:721 - 725.

[27] Dong L, Yang Q, Wu H, Xiao H, Xu M. High quality multi-spectral and panchromatic image fusion technologies based on curvelet transform, Neurocomputing, 2015; 159: 268 -274. 
[28] Wang D, Li Z, Dey N, Ashour AS, Moraru L, Biswas A, Shi F. Optical pressure sensors based plantar image segmenting using an improved fully convolutional network, Optik, 2019; 179, 99-114,

[29] Sung WT, Chang KY. Evidence-based multi-sensor information fusion for remote health care systems, Sensors and Actuators A: Physical, 2013; 204: 1 -19.

[30] Tian T, Sun S, Li N. Multi-sensor information fusion estimators for stochastic uncertain systems with correlated noises, Information Fusion, 2016; 27: 126 - 137.

[31] Liu J, Liu R, Wang Y, Chen J, Yang Y, Ma D. Image denoising searching similar blocks along edge directions, Signal Processing: Image Communication, 57, 33-45, 2017

[32] Hamid RS, Seyede MH. Image denoising in dual contourlet domain using hidden Markov tree models, Digital Signal Processing, 2017; 67:17-29.

[33] Liu X, Jing X, Tang G, Wu F, Ge Q. Image denoising using weighted nuclear norm minimization with multiple strategies, Signal Processing, 2017; 135, 239-252.

[34] Choudhury D, Naug A, Ghosh S. Texture and Color Feature Based WLS Framework Aided Skin Cancer Classification using MSVM and ELM, in Proc. IEEE INDICON, 2015; p.1-6.

[35] Al-Angari HM, Khandoker AH, Lee S., Almahmeed W., Safar KSA, Jelinek HF, Khalaf K, Novel dynamic peak and distribution plantar pressure measures on diabetic patients during walking, Gait \& Posture,2017; 51: 261-267.

[36] Wang M, Wang X, Fan Z, Chen F, Zhang S, Peng C, Research on feature extraction algorithm for plantar pressure image and gait analysis in stroke patients, Journal of Visual Communication and Image Representation, 2019; 58: 525-531.

[37] Wang D, Li Z, Dey N, Ashour AS, Moraru L, Biswas A, Shi F, Optical pressure sensors based plantar image segmenting using an improved fully convolutional network, Optik, 2019, 179: 99-114.

[38] Senthilkumar S, Geetha PC. Hidden Markov Model based channel selection framework for cognitive radio network Author links open overlay panel, Computers \& Electrical Engineering, 2018; 65:516-526.

[39] Huang S, Majid A, Sid-Ahmed MA. A hidden Markov model-based character extraction method, Pattern Recognition, 2008; 41: 2890 - 2900.

[40] Thanh N, Abbas K, Douglas C, Nahavandi S. Hidden Markov models for cancer classification using gene expression profiles, Information Sciences, 2015; 316: 293-307.

[41] Zhang P, Li M, Wu Y, Gan L, Liu M, Wang F, Liu G. Unsupervised multi-class segmentation of SAR images using fuzzy triplet Markov fields model, Pattern Recognition, 2012; 45: 4018-4033.

[42] Rozita R, Hossein P. Breast cancer detection using MRF-based probable texture feature and decisionlevel fusion-based classification using HMM on thermography images, Pattern Recognition, 2016; 51: 176186.

[43] Du J, Li W, Lu K, Xiao B. An overview of multi-modal medical image fusion, Neurocomputing, 2016; $215,3-20$.

[44] Niranjan PB, Chetty M, Kamruzzaman J. Hidden Markov Models Incorporating Fuzzy Measures and Integrals for Protein Sequence Identification and Alignment, Genomics, Proteomics \& Bioinformatics, 2008; 6(2): $98-110$.

[45] Xie F, Wu B, Hu Y, Wang Y, Jia G, Cheng Y. A generalized interval probability-based optimization method for training generalized hidden Markov model, Signal Processing, 2014; 94: 319-329.

[46] Zhao Q, Li X, Li Y, Zhao X. A fuzzy clustering image segmentation algorithm based on Hidden Markov Random Field models and Voronoi Tessellation, Pattern Recognition Letters, 2017; 85, 49-55.

[47] Rafiul H. A combination of hidden Markov model and fuzzy model for stock market forecasting, Neurocomputing, 2009; 72, 3439-3446.

[48] Tran TD, Michael W. Generalised Fuzzy Hidden Markov Models for Speech Recognition, Pal NR and Sugeno M (Eds.): In: Proc. AFSS 2002, LNAI 2275,2002, Springer, Berlin, Heidelberg.

[49] Harun U, Halife K. Classification of internal carotid artery Doppler signals using fuzzy discrete hidden Markov model, Expert Systems with Applications, 2011; 38: 7407-7414.

[50] Ferhat A, Yacine A, Abdelghani C, Mohammed S. Automatic Recognition of Gait Phases Using a Multiple-Regression Hidden Markov Model, IEEE/ASME Transactions on Mechatronics, 2018; 23(4):1597 $-1607$

[51] Juri T, Stefano R, Eduardo P, Patanè P, Cappa P. A Novel HMM Distributed Classifier for the Detection of Gait Phases by Means of a Wearable Inertial Sensor Network, Sensors, 2014; 14(9), 16212-16234.

[52] Pataky TC, Mu T, Bosch K, Rosenbaum D, Goulermas JY. Gait recognition: highly unique dynamic plantar pressure patterns among 104 individuals, Journal of the Royal Society Interface, 2012; 9(69): 790800 
[53] MathWorks Documentation Center. https://ww2.mathworks.cn/help/wavelet/ref/wfusimg.html

[54] Krista A, Yun Z, Peter D. Wavelet based image fusion techniques - An introduction, review and comparison ISPRS Journal of Photogrammetry and Remote Sensing, 2007; 62(4): 249-263

[55] Gleb Z, Alexey SM. Min-max optimal data encoding and fusion in sensor networks, Automatica, 2010; 46(9): 1546-1552.

[56] Li Z, He T, Cao L, Wu T, McCauley P, Balas VE, Shi F. Multi-source information fusion model in rulebased Gaussian-shaped fuzzy control inference system incorporating Gaussian density function, Journal of Intelligent \& Fuzzy Systems, 2015; 29: 2335-2344.

[57] Harun U, Emre EG, Uçman E, Saraçoğlu R. Biomedical system based on the Discrete Hidden Markov Model using the Rocchio-Genetic approach for the classification of internal carotid artery Doppler signals, Computer Methods and Programs in Biomedicine, 2011; 103(1): 51-60.

[58] Liu J, Wang Z, Xiao X. A hybrid SVM/DDBHMM decision fusion modeling for robust continuous digital speech recognition, Pattern Recognition Letters, 2007; 28(8): 912-920.

[59] Ioannis S, Steve P. An Introduction to Infinite HMMs for Single-Molecule Data Analysis, Biophysical Journal, 2017; 112(10): 2021-2029.

[60] Selma B, Clément C, Thierry P. Gesture sequence recognition with one shot learned CRF/HMM hybrid model, Image and Vision Computing, 2017; 61: 12-21.

[61] Zhao X, Zhang J, Chen Y, Li Q, Yang T, Pian C, Zhang L. Promoter recognition based on the maximum entropy hidden Markov model, Computers in Biology and Medicine, 2014; 51: 73-81.

[62] Duan Y, Liu F, Jiao L, Zhao P, Zhang L. SAR Image segmentation based on convolutional-wavelet neural network and markov random field, Pattern Recognition, 2017; 64: 255-267. 